\title{
On the Capacity of Multi-Channel Wireless Networks Using Directional Antennas
}

\author{
Hong-Ning Dai* ${ }^{*}$ Kam-Wing Ng* Raymond Chi-Wing Wong* and Min-You Wu ${ }^{\dagger}$ \\ *The Chinese University of Hong Kong, Hong Kong \\ \{hndai,kwng,cwwong\}@cse.cuhk.edu.hk \\ ${ }^{\dagger}$ Shanghai Jiao Tong University, China \\ wu-my@sjtu.edu.cn
}

\begin{abstract}
The capacity of wireless ad hoc networks is affected by two key factors: the interference among concurrent transmissions and the number of simultaneous transmissions on a single interface. Recent studies found that using multiple channels can separate concurrent transmissions and greatly improve network throughput. However, those studies only consider that wireless nodes are equipped with only omnidirectional antennas, which cause high collisions. On the other hand, some researchers found that directional antennas bring more benefits such as reduced interference and increased spatial reuse compared with omnidirectional antennas. But, they only focused on a single-channel network which only allows finite concurrent transmissions. Thus, combining the two technologies of multiple channels and directional antennas together potentially brings more benefits.

In this paper, we propose a multi-channel network architecture (called $M C-M D A$ ) that equips each wireless node with multiple directional antennas. We derive the capacity bounds of $M C-M D A$ networks under arbitrary and random placements. We will show that deploying directional antennas to multi-channel networks can greatly improve the network capacity due to increased network connectivity and reduced interference. We have also found that even a multi-channel network with a single directional antenna only at each node can give a significant improvement on the throughput capacity. Besides, using multiple channels mitigates interference caused by directional antennas. $M C$ - $M D A$ networks integrate benefits from multi-channel and directional antennas and thus have significant performance improvement.
\end{abstract}

\section{INTRODUCTION}

Wireless ad hoc networks typically consist of nodes that share one single channel for communications. It is found in [1] that in an ad hoc network with $n$ nodes under a random network ${ }^{1}$ placement, each node has a throughput capacity of $\Theta(1 / \sqrt{n \log n})$. Even under optimal arbitrary networks ${ }^{2}$, the network could only offer a per-node throughput of $\Theta(1 / \sqrt{n})$. The per-node throughput is decreased when the number of nodes increases. One major reason is that all the nodes within the network share the same medium. When a node transmits, its neighboring nodes are prohibited from transmitting due to interference. On the other hand, every node equipped with a single interface cannot transmit and receive at the same time (i.e., half-duplex mode). We call such single-channel networks using omnidirectional antennas as SC-Omni networks.

\footnotetext{
${ }^{1}$ In a random network, $n$ nodes are randomly placed, and the destination of a flow is also randomly chosen.

${ }^{2}$ In an arbitrary network, the location of nodes, and traffic patterns can be optimally controlled.
}

One approach to improve the network performance is to use multiple channels instead of using a single channel in a wireless network. The experimental results of [2]-[7] show that using multiple channels can significantly improve the network throughput. One possible reason is that multiple channels can separate multiple concurrent transmissions in frequency domain. Besides, a wireless node can be equipped with multiple network interfaces which allow multiple simultaneous transmissions/receptions to proceed at the same node. However, such networks in those studies [2]-[8] equip every node with omnidirectional antennas which have limited spatial reuse. Similarly, we name such multi-channel networks using multiple omnidirectional antennas as MC-MOmni networks.

Recent works such as [9]-[16] found that applying $d i$ rectional antennas instead of omnidirectional antennas to wireless networks can greatly improve the network capacity. For example, the analytical results in [9] show that using directional antenna in arbitrary networks achieves a capacity gain of $2 \pi / \sqrt{\alpha \beta}$ when both transmission and reception are directional, where $\alpha$ and $\beta$ are transmitter and receiver antenna beamwidths, respectively. Under random networks, the throughput improvement factor is $4 \pi^{2} /(\alpha \beta)$ for directional transmission and directional reception. Since the networks typically use one single channel only, we call such single channel networks using directional antennas as $S C$-DA networks.

Using directional antennas instead of omnidirectional antennas in a multi-channel wireless network is more beneficial. Therefore, we propose a novel network that integrates the two technologies. In this network, each node is equipped with multiple interfaces and each interface is associated with one directional antenna that can operate on different channels. Such multi-channel networks using multiple directional antennas are called as $M C-M D A$ networks that have the following characteristics.

- Each node is equipped with multiple network interface cards (NICs). Each NIC is mounted with a directional antenna.

- There are multiple non-overlapping channels available. Each antenna can switch to these channels quickly.

- All nodes can work in a full-duplex mode, in which a node can transmit and receive with different neighbors.

- Each node can communicate collision-freely and simultaneously with more than one node using different direc- 
tional antennas that operate on different channels.

Recently, DMesh [17] also proposed a similar architecture as ours. DMesh focuses on engineering issues of simulation and experimental studies about the throughput improvement. However, our work focuses on the theoretical analysis on the network capacity. Besides, our network is much more general, which can apply to wireless networks, but DMesh is limited to Wireless Mesh Networks. To the best of our knowledge, there is no theoretical analysis on the capacity of such networks. This paper concentrates on finding the capacity bounds for an $M C$-MDA network and exploring the benefits of this network.

The remainder of the paper is organized as follows. We have summarized our major contributions and outlined the main findings in Section II. Section III describes the antenna model and our proposed interference model, which will be used in our analysis. In Section IV, we present the analytical results of the transport capacity of arbitrary networks. Section V gives the analytical results of the throughput capacity of random networks. We summarize our work in Section VI.

\section{CONTRIBUtions AND MAIN RESUlts}

\section{A. Major contributions}

The primary research contributions of our paper can be summarized as follows.

1. We formally identify $M C-M D A$ networks that characterize the features of multi-channel wireless networks with multiple directional antennas at each node. The capacity of $M C$-MDA networks has not been studied before.

2. We derive the upper bounds on the capacity of $M C$ $M D A$ networks under arbitrary networks and random networks.

3. We also construct an arbitrary network and a random network, where both the lower bounds of the two networks have the same order of the upper bounds, which means that the derived upper bounds can be quite tight.

4. Our theoretical results show that integrating directional antennas with multi-channel networks can increase network connectivity and reduce interference, resulting in improved network capacity. Implications from the analytical results are also given.

Before presenting our main results, we need to give the assumptions and the notations first. We adopt the notations shown in Table I throughout this paper. In this paper, all nodes are equipped with the same type of antennas, which have the same beamwidth $\theta$ (generally less than $\pi$ ). Kyasanur and Vaidya [8] argued that the number of interfaces $m$ should not be greater than the number of channels $c$ (i.e., $1 \leq m \leq c$ ) because surplus interfaces are wasted if $m$ is greater than $c$. But, this condition is only valid when the networks adopt omnidirectional antennas. When directional antennas are used in the networks, this condition can be relaxed to that, in such networks, $m$ can be greater than $c$. More specifically, $m$ can be $\frac{2 \pi}{\theta} c$. With wider ranges of the number of interfaces, the deployment of antennas to a node is easier. Due to this additional property, we can achieve higher capacity in the
TABLE I

NOTATIONS USED IN THIS PAPER

\begin{tabular}{|c|l|}
\hline$n$ & the number of nodes \\
\hline$c$ & the number of available channels \\
\hline$m$ & $\begin{array}{l}\text { the number of interfaces at each node where each } \\
\text { interface is associated with a directional antenna }\end{array}$ \\
\hline$(m, c)$-network & $\begin{array}{l}\text { a network with } c \text { channels and } m \text { directional } \\
\text { antennas per node }\end{array}$ \\
\hline$W$ & $\begin{array}{l}\text { the total data rate by using all channels. Each } \\
\text { channel can support the data rate } W / c\end{array}$ \\
\hline$\theta$ & the beamwidth of a directional antenna \\
\hline$\lambda$ & each node sends $\lambda$ bits per second \\
\hline$M I N_{O}(f(n), g(n))$ & is equal to $f(n)$, if $f(n)=O(g(n))$, else it is equal to $g(n)$ \\
\hline
\end{tabular}

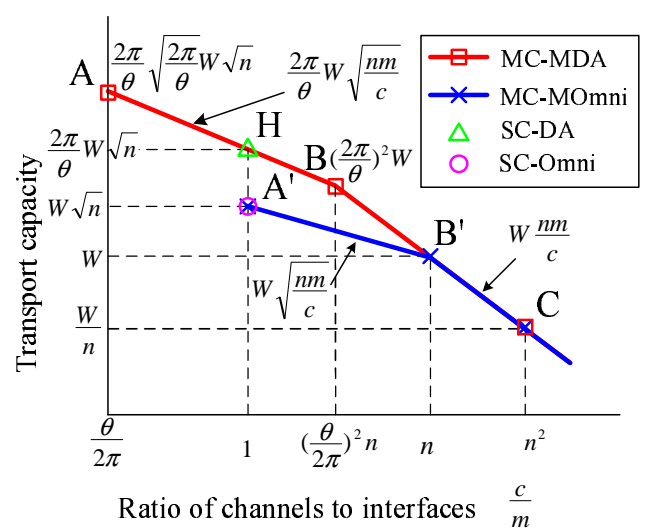

Fig. 1. The capacity regions under different $\frac{c}{m}$ in arbitrary networks (figure is not to scale)

networks. Detailed discussion will be given in Section II-B.3.

\section{B. Summary of results}

Since the capacity of an $M C$ - $M D A$ network depends on the ratio of $\frac{c}{m}$, we present the results according to the ratio of $\frac{c}{m}$.

1. Results for Arbitrary Networks

As shown in Fig. 1, the transport capacity ${ }^{3}$ of an $(m, c)$ network has two regions as follows according to ratio of $c$ to $m$ (from Theorem 2 and Theorem 3).

1) When $\frac{c}{m}$ is $O\left(\left(\frac{\theta}{2 \pi}\right)^{2} n\right)$, the transport capacity is $\Theta\left(\frac{W}{\theta} \sqrt{\frac{m m}{c}}\right)$ (which is $\frac{2 \pi}{\theta} W \sqrt{\frac{n m}{c}}$ ) bit-meters/sec (segment A-B in Fig. 1) with a capacity gain of $\frac{2 \pi}{\theta}$ over an MC-MOmni network (segment $\mathrm{A}^{\prime}-\mathrm{B}^{\prime}$ ).

2) When $\frac{c}{m}$ is $\Omega\left(\left(\frac{\theta}{2 \pi}\right)^{2} n\right)$, the transport capacity is $\Theta\left(W \frac{n m}{c}\right)$ bit-meters/sec (segment B-C in Fig. 1), which is independent of beamwidth $\theta$.

2. Results for Random Networks

As shown in Fig. 2, the throughput capacity ${ }^{4}$ of an $(m, c)$ network has three regions as follows according to ratio of $c$ to $m$ (from Theorem 4 and Theorem 5).

1) When $\frac{c}{m}$ is $O\left(\left(\frac{\theta}{2 \pi}\right)^{2} \log n\right)$, the throughput capacity is $\Theta\left(\frac{W}{\theta^{2}} \sqrt{\frac{n}{\log n}}\right)$ (which is $\left.\frac{4 \pi^{2}}{\theta^{2}} W \sqrt{\frac{n}{\log n}}\right)$ bits/sec (segment

\footnotetext{
${ }^{3}$ the transport capacity is that the network transport one bit-meter per second when one bit has been transported a distance of one meter within one second

${ }^{4}$ We just consider the aggregate throughput capacity of the whole network, which is measured in terms of bits/sec.
} 


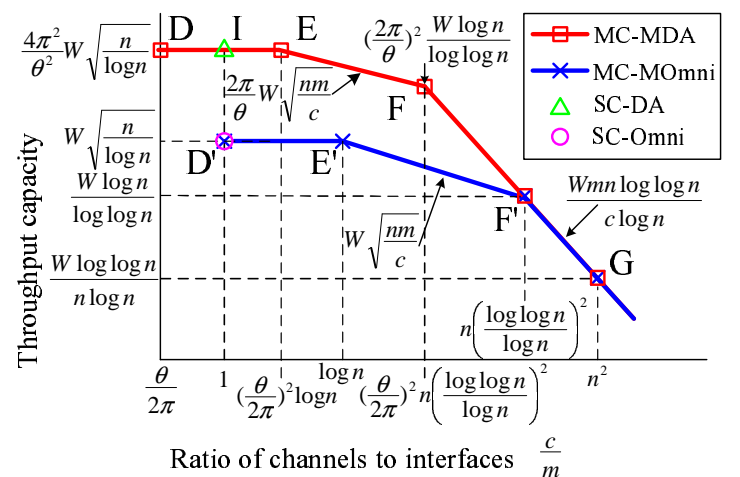

Fig. 2. The capacity regions under different $\frac{c}{m}$ in random networks (figure is not to scale)

D-E in Fig. 2) with a capacity gain $\frac{4 \pi^{2}}{\theta^{2}}$ over an $M C$ MOmni network (segment $\mathrm{D}^{\prime}$-E' $)$.

2) When $\frac{c}{m}$ is $\Omega\left(\left(\frac{\theta}{2 \pi}\right)^{2} \log n\right)$ and also $O\left(\left(\frac{\theta}{2 \pi}\right)^{2} n\left(\frac{\log \log n}{\log n}\right)^{2}\right)$, the throughput capacity is $\Theta\left(\frac{W}{\theta} \sqrt{\frac{n m}{c}}\right)$ (which is $\frac{2 \pi}{\theta} W \sqrt{\frac{n m}{c}}$ ) bits/sec (segment E-F in Fig. 2), and the capacity gain over an MC-MOmni network is $\frac{2 \pi}{\theta}$ (segment $\mathrm{E}^{\prime}-\mathrm{F}^{\prime}$ ).

3) When $\frac{c}{m}$ is $\Omega\left(\left(\frac{\theta}{2 \pi}\right)^{2} n\left(\frac{\log \log n}{\log n}\right)^{2}\right)$, the throughput capacity is $\Theta\left(\frac{W m n \log \log n}{c \log n}\right)$ bits/sec (segment F-G in Fig. 2), which is independent of beamwidth $\theta$.

\section{Comparisons with Other Networks}

We consider an arbitrary network when $c=m=1$, which has a capacity of $\frac{2 \pi}{\theta} W \sqrt{n}$ (point $\mathrm{H}$ in Fig. 1). Such network is an $S C$-DA network, which can be regarded as a special case of an $M C-M D A$ network. Similarly, an SC-Omni network is a special case of an MC-MOmni network when $c=m=1$ (point $\mathrm{A}^{\prime}$ in Fig. 1). When $\frac{c}{m}$ is $O\left(\left(\frac{\theta}{2 \pi}\right)^{2} n\right)$, the capacity of an $M C-M D A$ network is mainly affected by the interference. The interference can be mitigated by assigning concurrent transmissions under difference channels. Thus, if the number of interfaces $m$ is fixed, increasing the number of channels $c$ is helpful to reduce the interference. Specifically, when the number of channels is $\left(\frac{\theta}{2 \pi}\right)^{2} n m$, (i.e., point $\mathrm{B}$ in Fig. 1), all transmission can be regarded as collision-free. However, when the number of channels is increased further and $\frac{c}{m}$ is $\Omega\left(\left(\frac{\theta}{2 \pi}\right)^{2} n\right)$, the capacity is affected by the interface constraint. The capacity drops even faster when the ratio of $\frac{c}{m}$ increases since the capacity is inverse-proportional to the ratio of $\frac{c}{m}$. Besides, from Fig. 1, an $M C-M D A$ network requires less channels to reach the collision-free condition (at point $\mathrm{B})$, compared with an $M C-M O m n i$ network (at point $\mathrm{B}^{\prime}$ ).

When a random network is considered, an $S C$ - $D A$ network is a special case of an $M C-M D A$ network when $c=m=1$ (point I in Fig. 2). And an SC-Omni network is a special case of MC-MOmni networks when $c=m=1$ (point $\mathrm{D}^{\prime}$ in Fig. 2). In a random placement, an $M C-M D A$ network has a capacity gain of $\frac{4 \pi^{2}}{\theta^{2}}$ over an MC-MOmni network if $\frac{c}{m}$ is $O\left(\left(\frac{\theta}{2 \pi}\right)^{2} \log n\right)$. The reason is that directional antennas can greatly improve the network connectivity. Since using directional antennas can reduce interference, an $M C-M D A$ network has a capacity gain of $\frac{2 \pi}{\theta}$ over an MC-MOmni network when $\frac{c}{m}$ is $\Omega\left(\left(\frac{\theta}{2 \pi}\right)^{2} \log n\right)$ and also $O\left(\left(\frac{\theta}{2 \pi}\right)^{2} n\left(\frac{\log \log n}{\log n}\right)^{2}\right)$. When $\frac{c}{m}$ is $\Omega\left(\left(\frac{\theta}{2 \pi}\right)^{2} n\left(\frac{\log \log n}{\log n}\right)^{2}\right)$, similar to an MC-MOmni network, the capacity of an $M C-M D A$ network is only affected by the flow bottleneck in a node.

$M C-M D A$ networks are promising to improve the network capacity. Since directional antennas can greatly increase the spatial reuse, the same channels can be reused in different directions without collisions, but omnidirectional antennas cannot. So, the number of interfaces $m$ can be greater than the number of channels $c$. The maximum number of antennas on a node in an $M C-M D A$ network can be $\frac{2 \pi}{\theta} c$. However, in an $M C$-MOmni network, $m$ is always not greater than $c$ [8]. Thus, in an arbitrary placement, an $M C$-MOmni network has at most a capacity bounded by $\Theta(W \sqrt{n})$. When $m$ has the maximum value $\frac{2 \pi}{\theta} c$, an $M C-M D A$ network can have a capacity gain $\frac{2 \pi}{\theta} \sqrt{\frac{2 \pi}{\theta}}$ over an MC-MOmni network. For example, when $\theta$ is $\frac{\pi}{4}$ and $c$ is 3 , the maximum number of interfaces $m$ is 24 . Then, we have the capacity gain $16 \sqrt{2}$ (nearly 23 ) times over an MC-MOmni network which has the same number of channels $(c=3)$ and 3 interfaces at each node.

But, the number of antennas should not be set too large. One major reason is that a single interface can only share the capacity gain of $\frac{2 \pi}{\theta} \sqrt{\frac{1}{m c}}$, which decreases when the number of interfaces increases. Let us consider the same example mentioned above for illustration. A single interface can share a capacity gain only $4 / \sqrt{6}$. The number of antennas is also limited by the size and cost of antennas. Choosing the number of antennas needs considering some engineering issues such as the device cost, the size of antennas and the interferences among the antennas. However, our work just focuses on theoretical performance analysis. How to choose the proper number of interfaces is our future work.

\section{MODEL}

In an $M C-M D A$ network, each node is equipped with $m$ directional antennas that can be approximated by the following antenna model. Besides, since interference among concurrent transmissions is a major reason affecting the network capacity, we propose a receiver-based interference model and derive the condition that a transmission is successful.

\section{A. Antenna Model}

In this paper, we consider a directional antenna model that is used in previous works [9], [13]-[15]. Sidelobes and backlobes are ignored in this model. The reasons why we simplify the model are summarized as follows. First, even in a more realistic model, the sidelobes are too small to be ignored. For example, the main gain is more than 100 times of the gain of sidelobes when the main beamwidth is less than $40^{\circ}$ in the cone-sphere model [10]. Secondly, smart antennas often have null capability that can almost eliminate the sidelobes and backlobes. Ref. [18] derives the impact of null capability of smart antennas on the network capacity. More complexed antenna models will be considered in the future work. 


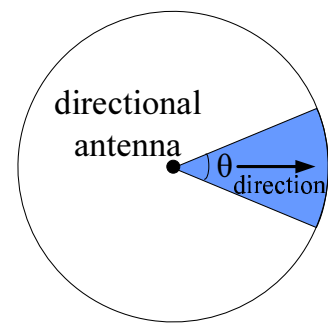

Fig. 3. The Antenna Model

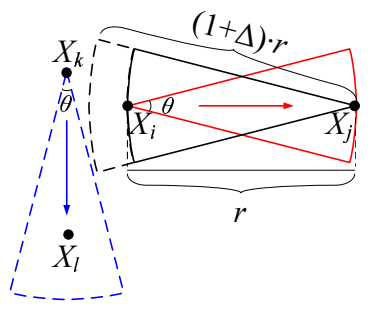

Fig. 4. The Receiver-based Interference Model
Our proposed model assumes that a directional antenna gain is within a specific angle $\theta$, where $\theta$ is the beamwidth of the antenna. The gain outside the beamwidth is assumed to be zero. At any time, the antenna beam can only be pointed to a certain direction, as shown in Fig. 3, in which the antenna is pointing to the right. Thus, the probability that the beam is switched to cover each direction is $\theta / 2 \pi$.

\section{B. Receiver-based Interference Model}

Based on the protocol model in [1], we propose a receiverbased interference model with extensions of directional antennas. Our model only considers directional transmission and directional reception, which can maximize the benefits of directional antennas.

If node $X_{i}$ transmits to node $X_{j}$ over a channel, the transmission is successfully completed by node $X_{j}$ if no nodes within the region covered by $X_{j}$ 's antenna beam will interfere with $X_{j}$ 's reception. Therefore, for every other node $X_{k}$ simultaneously transmitting over the same channel, and the guard zone $\Delta>0$, the following condition holds.

$$
\left\{\begin{array}{l}
\left|X_{k}-X_{j}\right| \geq(1+\Delta)\left|X_{i}-X_{j}\right| \\
\text { or } X_{k} \text {,s beam does not cover node } X_{j}
\end{array}\right.
$$

where $X_{i}$ not only denotes the location of a node but refers to the node itself. In this model, each node is equipped with one single directional antenna that can operate over $c$ channels. Fig. 4 shows that a transmission from node $X_{k}$ will not cause interference to $X_{i}$ 's transmission since the antenna beam of $X_{k}$ does not cover receiver $X_{j}$.

Gupta and Kumar [1] established a physical model in which the success probability of a transmission is related to the Signal-to-Interference-Noise Ratio (SINR). When the fading factor is greater than two (it is common in a real world), the physical model is equivalent to the interference model. Thus, we will only consider the interference model in this paper.

\section{TRANSPORT CAPACITY FOR ARbitrary Networks}

Since the capacity of an $M C-M D A$ network is affected by two factors, i.e., the interference among concurrent transmissions and the number of simultaneous transmissions on an interface, we derive different upper bounds when considering these two factors, respectively in Section IV-A. To illustrate that the upper bounds are quite tight, we construct a network that can achieve the lower bounds having the same order of the upper bounds in Section IV-B.

\section{A. Upper Bound}

Similar to an MC-MOmni network [8], the transport capacity of an $M C-M D A$ network is also limited by by two constraints: interference constraint and interface constraint.

(1) Interference Constraint: the interference around a receiver is affected by the number of interfering nodes in its neighborhood, which is determined by the size of the interference region. When we use directional antennas at both transmitter and receiver ends, the condition interference zone is $\frac{\theta^{2}}{(2 \pi)^{2}}$ portion of that when omnidirectional antennas are used at both ends [9]. We derive the first bound when considering the interference constraint and have the following theorem.

Theorem 1: The capacity of a multi-channel network equipped with $m$ directional antennas is $O\left(\frac{W}{\theta} \sqrt{\frac{n m}{c}}\right)$ bitmeters/sec. Compared to a multi-channel network using $m$ omnidirectional antennas per node, the capacity gain is $\frac{2 \pi}{\theta}$.

Proof: We present a proof of the bound in Appendix A.

It is proved in [8] that the capacity of an MC-MOmni $(m, c)$ network is bounded by $\frac{W}{\Delta} \sqrt{\frac{2 n m}{\pi c}}$. Compared with this result, an $M C$-MDA $(m, c)$-network has a capacity gain of $\frac{2 \pi}{\theta}$.

(2) Interface Constraint: we consider the interface constraint of an $M C$ - $M D A$ network. Since every node has $m$ interfaces, there are $m n$ interfaces in the whole network. Each interface can support at most $\frac{W}{c}$ bits/sec and the maximum distance that a bit can travel in the network is $\Theta(1)$ meters. Thus, the interface bound of the network is $O\left(W \frac{n m}{c}\right)$ bit-meters/sec.

Combining the two constraints, the network transport capacity is $O\left(M I N_{O}\left(\frac{W}{\theta} \sqrt{\frac{n m}{c}}, W \frac{n m}{c}\right)\right)$ bit-meters/sec. The minimum bound of them is an upper bound on the network capacity. Then, we have the following theorem on the transport capacity of an arbitrary network.

Theorem 2: The upper bound on the transport capacity of an $(m, c)$-network is shown as follows.

i) When $\frac{c}{m}$ is $O\left(\left(\frac{\theta}{2 \pi}\right)^{2} n\right)$, the transport capacity is $O\left(\frac{W}{\theta} \sqrt{\frac{m m}{c}}\right)$ bit-meters/sec.

ii) When $\frac{c}{m}$ is $\Omega\left(\left(\frac{\theta}{2 \pi}\right)^{2} n\right)$, the transport capacity is $O\left(W \frac{n m}{c}\right)$ bit-meters/sec.

The network capacity of a $(1,1)$ network is $O\left(\frac{W}{\theta} \sqrt{n}\right)$ bitmeter/sec, which matches the result obtained by [9]. Thus, an $S C$-DA can be regarded as a special case of an $M C$ $M D A$ network when $m=c=1$. When $\frac{c}{m}$ is $O\left(\left(\frac{\theta}{2 \pi}\right)^{2} n\right)$, an $M C$ - $M D A$ network has a capacity gain $\frac{2 \pi}{\theta}$ over an $M C$ MOmni network, and a capacity gain $\frac{2 \pi}{\theta} \sqrt{\frac{m}{c}}$ over an SC-Omni network, where $m$ can be greater than $c$.

\section{B. Constructive Lower Bound}

In this section, we construct a network that can achieve the capacity of $\Omega\left(M I N_{O}\left(\frac{W}{\theta} \sqrt{\frac{n m}{c}}, W \frac{n m}{c}\right)\right)$ bit-meters/sec in order to show that the upper bound derived in Section IV-A is tight. First, we divide the unit-area into a number of equal-sized cells and each cell has the same number of nodes. In each cell, to ensure collision-free transmissions, we separate transmitters and receivers at different positions and their antenna beams are aimed to proper directions. For example, all transmitters adjust their antennas to $30^{\circ}$ east of 


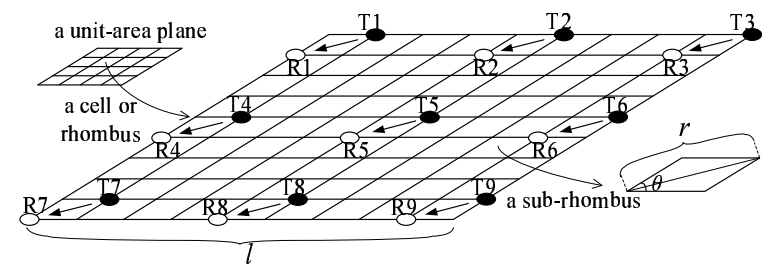

Fig. 5. A possible placement of nodes within a cell

due north and all receivers point their receiving antennas to $30^{\circ}$ west of due south. Then, we illustrate that this placement can guarantee correct transmissions for all communicating pairs. Using the result in [8], we can extend the result of a $(1, c)$-network to that of an $(m, c)$-network. This lemma also holds for a network using directional antennas instead.

Lemma 1: [8] An $(m, c)$-network can support at least half of the capacity supported by a $\left(1,\left\lfloor\frac{c}{m}\right]\right)$ network.

We exhibit a scenario where the lower bound is achieved.

(1) Each node is equipped with a directional antenna with beamwidth $\theta$. Let $g=\min \left(\frac{2 \pi c}{\theta}, \frac{n \theta}{36 \pi}\right)$, which will be used to calculate the capacity of the network. We divide the unit-area plane into $\frac{n \theta}{36 \pi g}$ equal-sized rhombi. Thus, each cell has $\frac{36 \pi g}{\theta}$ nodes. Since the total area is 1 , each cell has a size of $\frac{36 \pi g}{n \theta}$. All sides of every rhombus has a length $l=\sqrt{\frac{36 \pi g}{n \theta \sin \theta}}$.

(2) As shown in Fig. 5, we further divide every cell into $\left(\frac{2 \pi}{\theta}+1\right)^{2}$ equal-sized sub-rhombi and place $\frac{36 \pi g}{\theta}$ nodes into 18 positions, which are divided into two groups, namely (A) R1,R2,..,R9 (white dots in Fig. 5) and (B) T1,T2,..,T9 (black dots in Fig. 5). So there are $\frac{2 \pi g}{\theta}$ nodes in each position. Nodes that are placed at group (A) play as receivers and those placed at group (B) act as transmitters. Two neighboring transmitters are separated at least $\frac{2 \pi}{\theta}$ times side-length of a sub-rhombus. After some derivations, we obtain the transmission range $(r$ is the diagonal length (see Fig. 5)) $r=\frac{1}{\frac{2 \pi}{a}+1} \sqrt{\frac{72 \pi g(1+\cos \theta)}{n \theta \sin \theta}}$. Here, $\frac{2 \pi}{\theta}, \sin \theta$ and $\cos \theta$ can be regarded as constants. So $r=k_{1} \sqrt{\frac{2 \pi g}{n \theta}}$, where $k_{1}$ is a constant.

(3) Consider a pair of communicating nodes $X_{i}$ and $X_{j}$ that are located in T5 and R5, respectively. The $X_{i}$ 's antenna is adjusted to face $X_{j}$, and the antenna of $X_{j}$ is pointed to $X_{i}$ as well. Thus, $X_{j}$ is only affected by the nodes that are in the same line as $X_{i}$. From Fig. 5, the nearest interfering nodes within the cell, other than those located in T5, must be located in T3, which is at least a distance of $r(1+\Delta)$ away from $X_{j}$ (where $\Delta=\frac{2 \pi}{\theta}>0$ ). Thus, under the interference model Eq. (1), the transmission between nodes $X_{i}$ and $X_{j}$ is not affected by other transmissions in the network, and this result holds for all communicating pairs.

In a $(1, c)$-network, there are at most $n / 2$ pairs of nodes that can transmit. Each pair transmits at a rate of $W / c$ over a distance $r$. Hence, the total transport capacity of the network is not greater than $\frac{n}{2} \frac{W}{c} r=\frac{n}{2} \frac{W}{c} k_{1} \sqrt{2 \pi g /(n \theta)}$. Recall $g=\min \left(\frac{2 \pi c}{\theta}, \frac{n \theta}{36 \pi}\right)$. Thus, total capacity is bounded by $\frac{k_{1}}{2} \frac{2 \pi}{\theta} W \sqrt{\frac{n}{c}}$ if $g=\frac{2 \pi c}{\theta}$, otherwise it is bounded by $\frac{k_{1}}{6 \sqrt{2}} W \frac{n}{c}$ if $g=\frac{n \theta}{36 \pi}$.
Hence, the capacity of a $(1, c)$-network to be $\Omega\left(M I N_{O}\left(\frac{W}{\theta} \sqrt{\frac{n}{c}}, W \frac{n}{c}\right)\right) \quad$ bit-meters/sec. By Lemma 1 , we extend the result from a $(1, c)$-network to an $(m, c)$-network. Thus, the capacity of an $(m, c)$-network is $\quad \Omega\left(M I N_{O}\left(\frac{W}{\theta} \sqrt{\frac{n}{\left[\frac{c}{m}\right]}}, W \frac{n}{\left[\frac{c}{m}\right]}\right)\right) \quad$ bit-meters/sec. As $\frac{1}{\left.\frac{c}{m}\right]} \geq \frac{1}{\frac{c}{m}}$, the capacity is $\Omega\left(M I N_{O}\left(\frac{W}{\theta} \sqrt{\frac{m n}{c}}, W \frac{m n}{c}\right)\right)$.

The lower bound has the same order of the upper bound. Thus, the upper bounds that we obtained in Section IV-A are tight. So we have a theorem on the achievable capacity.

Theorem 3: There is a placement of nodes and an assignment of traffic patterns such that.

i) When $\frac{c}{m}$ is $O\left(\left(\frac{\theta}{2 \pi}\right)^{2} n\right)$, the transport capacity is $\Omega\left(\frac{W}{\theta} \sqrt{\frac{n m}{c}}\right)$ bit-meters/sec.

ii) When $\frac{c}{m}$ is $\Omega\left(\left(\frac{\theta}{2 \pi}\right)^{2} n\right)$, the transport capacity is $\Omega\left(W \frac{n m}{c}\right)$ bit-meters/sec.

\section{Some Possible Implications}

Using directional antennas to multi-channel network is beneficial to to improve the network capacity. Directional antennas can separate multiple concurrent transmissions and increase spatial reuse. A small number of channels can be reused in different directions without collisions. So, the number of interfaces $m$ can be greater than the number of channels $c$, which is different from the results in [8]. Since $m$ has the maximum value $\frac{2 \pi}{\theta} c$, an $M C-M D A$ network can have a capacity gain $\frac{2 \pi}{\theta} \sqrt{\frac{2 \pi}{\theta}}$, which has a significant increment over an MC-MOmni network. But, the number of antennas should not be set too large. A single interface can only share the capacity gain of $\frac{2 \pi}{\theta} \sqrt{\frac{1}{m c}}$, which is decreasing when the number of interfaces increases. The number of antennas is also limited by the size and cost of antennas. There is a trade-off between the number of antennas and the cost.

With decreasing the beamwidth $\theta$, the capacity is growing fastly. However, the capacity will not grow arbitrarily high when the beamwidth decreases further and even approaches to zero. Yi et al. [9] have observed that when the beamwidth is too small, the interference has been fully reduced and there is no any further improvement by decreasing the beamwidth of the antennas. Actually, when the beamwidth is narrow enough (more specially, less than a certain angle) a transmission can yield a high success probability. That is, the transmission can be regarded as collision-tolerant [19]. It is observed that if the beamwidth is less than $\frac{\pi}{12}$ (i.e., $15^{\circ}$ ) and nodes are not densely distributed and both directional antennas are used at the transmitter and the receiver, then the probability of a successful transmission is greater than $99 \%$.

\section{Throughput CAPACITY FOR RANDOM NeTwORKS}

Different from arbitrary networks, the capacity of random networks is affected by three major factors [8]: network connectivity, interference, and destination bottleneck. So we derive different upper bounds under different factors in Section V-A. We evaluate random networks with throughput capacity instead of transport capacity because throughput capacity is 
commonly used to evaluate random networks (e.g., [1], [8] and [9]). In order to prove that the upper bounds are quite tight, in Section V-B, we construct a network that can achieve the lower bounds having the same order of the upper bounds.

\section{A. Upper Bound}

As we mentioned before, the capacity of multi-channel random networks using directional antennas is limited by the following three constraints [8].

1) Connectivity constraint: When we say a network is connected, we mean that a network is connected $w h p^{5}$. This constraint is necessary for a random network to ensure that the network is connected. When each node is equipped with directional antennas in a random network, a high connectivity can be gained. Previous work [9] found that the upper bound of a random network using directional antennas at both the transmitter and the receiver is $O\left(\frac{W}{\theta^{2}} \sqrt{\frac{n}{\log n}}\right) \mathrm{bits} / \mathrm{sec}$. This bound is also applicable to $M C-M D A$ networks.

2) Interference constraint: The capacity of multi-channel random networks using directional antennas is also constrained by interference. Thus, similar to arbitrary networks, by Theorem 1, a random network with $m$ directional antennas have $O\left(\frac{W}{\theta} \sqrt{\frac{n m}{c}}\right)$ bit-meters/sec. Since each pair of sourcedestination in a random network is separated by a distance of $\Theta(1)$ meter on average, the network capacity of random networks is at most $O\left(\frac{W}{\theta} \sqrt{\frac{n m}{c}}\right) \mathrm{bit} / \mathrm{sec}$.

3) Destination bottleneck constraint: The capacity of a multi-channel network is restricted by the flows ${ }^{6}$ toward a destination node. Before calculating the upper bound under bottleneck constraint, we need to bound the maximum number of flows for a destination node first.

In a random network, a node randomly chooses its destination. Thus, it is possible that a node assembles multiple flows. Let $F(n)$ be the maximum number of flows for a destination node. The process of choosing a destination node can be regarded as randomly throwing a ball into a bin, which is similar to [20]. Hence, we use the result of [20] and have Lemma 2 to bound the maximum number of flows for a destination node.

Lemma 2: The maximum number of flows $F(n)$ from other nodes to a chosen destination is $\Theta\left(\frac{\log n}{\log \log n}\right)$, whp.

In an $(m, c)$-network, each channel supports a maximum data rate of $\frac{W}{c}$ bits/sec. Suppose that node $X_{l}$ that is the destination of the maximum number of flows $F(n)$. Hence, the total data rate at node $X_{l}$ with $m$ antennas is $\frac{W m}{c}$ bits/sec. Since node $X_{l}$ has $F(n)$ incoming flows, the data rate of the flow with the minimum rate is at most $\frac{W m}{c F(n)} \mathrm{bits} / \mathrm{sec}$. Hence, the minimum per-node throughput capacity is not greater than $\frac{W m}{c F(n)}$, which implies that the network capacity is at most $O\left(\frac{W m n}{c F(n)}\right)$ bits/sec. Substituting $F(n)$ by Lemma 2, the network capacity is at most $O\left(\frac{W m n \log \log n}{c \log n}\right)$ bits/sec.

Combining the three bounds under the three constraints, we obtain that the network capacity is at

\footnotetext{
${ }^{5}$ In this paper, whp means with probability $\geq 1-1 / n$

${ }^{6}$ The traffic from a source node to a destination node is called a flow.
}

most $O\left(M I N_{O}\left(\frac{W}{\theta^{2}} \sqrt{\frac{n}{\log n}}, \frac{W}{\theta} \sqrt{\frac{n m}{c}}, \frac{W m n \log \log n}{c \log n}\right)\right)$ bits/sec. Thus, we have the following theorem on the upper bound on the capacity of random networks.

Theorem 4: The upper bound on the capacity of a random network is as follows.

1) When $\frac{c}{m}$ is $O\left(\left(\frac{\theta}{2 \pi}\right)^{2} \log n\right)$, the throughput capacity is $O\left(\frac{W}{\theta^{2}} \sqrt{\frac{n}{\log n}}\right)$ bits/sec with a capacity gain of $\frac{4 \pi^{2}}{\theta^{2}}$ over an MC-MOmni network.

2) When $\frac{c}{m}$ is $\Omega\left(\left(\frac{\theta}{2 \pi}\right)^{2} \log n\right)$ and also $O\left(\left(\frac{\theta}{2 \pi}\right)^{2} n\left(\frac{\log \log n}{\log n}\right)^{2}\right)$, the throughput capacity is $O\left(\frac{W}{\theta} \sqrt{\frac{n m}{c}}\right)$ bits/sec with a capacity gain of $\frac{2 \pi}{\theta}$ over an MC-MOmni network.

3) When $\frac{c}{m}$ is $\Omega\left(\left(\frac{\theta}{2 \pi}\right)^{2} n\left(\frac{\log \log n}{\log n}\right)^{2}\right)$, the throughput capacity is $O\left(\frac{W m n \log \log n}{c \log n}\right) \mathrm{bits} / \mathrm{sec}$.

When $\frac{c}{m}$ is $O\left(\left(\frac{\theta}{2 \pi}\right)^{2} \log n\right)$, an MC-MDA network has a capacity gain $4 \pi^{2} / \theta^{2}$ over an $M C$-MOmni network. The reason is that directional antennas greatly improve the network connectivity. Similar to MC-MOmni networks, the ratio of $c$ to $m$ has no impact on the network capacity. When $\frac{c}{m}$ is $\Omega\left(\left(\frac{\theta}{2 \pi}\right)^{2} \log n\right)$ and also $O\left(\left(\frac{\theta}{2 \pi}\right)^{2} n\left(\frac{\log \log n}{\log n}\right)^{2}\right)$, the capacity of an $M C-M D A$ network is $O\left(\frac{W}{\theta} \sqrt{\frac{n m}{c}}\right)$, which has a capacity gain of $\frac{2 \pi}{\theta}$ over an MC-MOmni network. When $\frac{c}{m}$ is $\Omega\left(\left(\frac{\theta}{2 \pi}\right)^{2} n\left(\frac{\log \log n}{\log n}\right)^{2}\right)$, the capacity of an $M C-M D A$ is $O\left(\frac{W m n \log \log n}{c \log n}\right)$, which is the same as an $M C$-MOmni network.

An $S C$-DA network can be regarded as a special case of an $M C$ - $M D A$ network when $c=m=1$. When $c=m=1$, the capacity of an $S C-D A$ network can fall into $O\left(\frac{W}{\theta^{2}} \sqrt{\frac{n}{\log n}}\right)$ or $O\left(\frac{W}{\theta} \sqrt{\frac{n m}{c}}\right)$, which is related to $\log n(\log n \geq 1$ or $\leq 1)$. Similarly, an SC-Omni network can be regarded as a specific case of an MC-MOmni network, when $c=m=1$.

\section{B. Constructive Lower Bound}

To prove that the upper bound in Section V-A can be quite tight, we begin to construct a network and then design a routing scheme and a transmission scheduling mechanism as follows. Step 1 (Torus Division): we divide the unit-area plane into even-sized squares. The size of each square suffices three constraints mentioned previously. Step 2 (Routing Construction): we design a routing scheme that assigns a flow to a node with balanced flows at each node. In the following, we will find that the total flows assigned to any node is only determined by the square size. Step 3 (Transmission Scheduling): we consider a $(1, c)$-network. To ensure the network satisfies two additional constraints (which was used in [8] and will be described in details later), we propose a transmission scheduling mechanism to ensure a collision-free transmission within that channel. Finally, we obtain the capacity of a $(1, c)$ network. Using Lemma 1 mentioned in Section IV-B (which also holds for a random network using directional antennas), we extend the result to an $(m, c)$-network and obtain the constructive lower bound.

Step 1 (Torus Division): We divide the unit-area plane into equal-sized squares. The size of each square denoted by $a(n)$ must satisfy the three constraints mentioned in Section V-A. 
It is found in [8] that when the size of each square is greater than a certain value, each square must contain a certain number of nodes. So, it can guarantee successful transmissions from source nodes to destination nodes. We state their lemma here.

Lemma 3: [8] If $a(n)$ is greater than $\frac{50 \log n}{n}$, each cell has $\Theta(n a(n))$ nodes per cell, whp.

To simplify the calculation, we take $\frac{100 \log n}{n}$ for a large $n$.

It is found in [9] that in a random network, using directional antennas at both the transmitter and the receiver can reduce the interfering area by $\left(\frac{\theta}{2 \pi}\right)^{2}$. Since the number of nodes is proportional to the size of the area, the number of interfering nodes is reduced by $\left(\frac{\theta}{2 \pi}\right)^{2}$. In other words, the interferencetolerant capability of a node is increased by $\left(\frac{\theta}{2 \pi}\right)^{2}$. Thus, for a $(1, c)$-network, $a(n)$ is equal to $\max \left(\frac{100 \log n}{n}, \frac{c}{n}\left(\frac{\theta}{2 \pi}\right)^{2}\right)$.

To ensure the flow bottleneck constraint, we take $\left(\frac{1}{F(n)}\right)^{2}$ as another possible value for $a(n)$, where $F(n)=\Theta\left(\frac{\log n}{\log \log n}\right)$ (by Lemma 2). Then, we have

$$
a(n)=\min \left(\max \left(\frac{100 \log n}{n}, \frac{c}{n}\left(\frac{\theta}{2 \pi}\right)^{2}\right),\left(\frac{1}{F(n)}\right)^{2}\right)
$$

If a node in cell $\mathrm{B}$ interferes with another transmission in cell $\mathrm{A}$, this cell is called an interfering cell. We prove that the number of interfering cells around a cell is a constant, which is independent of $a(n)$ and $n$. Thus, we have Lemma 4 .

Lemma 4: The number of cells that interfere with any given cell is bounded by a constant $k_{2}$ (where $k_{2}=81(2+\Delta)^{2} \frac{\theta^{2}}{4 \pi^{2}}$ ), which is independent of $a(n)$ and $n$.

Proof: The detailed proof is stated in Appendix B.

Step 2 (Routing Construction): We construct a simple routing scheme that chooses a route with the shortest distance to forward packets. A straight line denoted by S-D line is passing through the cells that source node $S$ and destination node $D$ are located. Packets are delivered along the cells lying on the source-destination line. Then, we choose a node within each cell lying on the straight line to carry that flow. The node assignment is based on load balancing. The flow assignment procedure is divided into two sub-steps.

Step 2(a): source and destination nodes are assigned. For any flow that originates from a cell, source node $\mathrm{S}$ is assigned to the flow. Similarly, for any flow that terminates in a cell, destination node D is assigned to the flow. After this step, only those flows passing through a cell (not originating or terminating) are left. Step 2(b): we assign the remaining flows. To balance the load, we assign each remaining flow to a node that has the least number of flows assigned to it. Thus, each node has nearly the same number of flows.

It is found in [21] that the number of S-D lines passing through any cell is $O(n \sqrt{a(n)})$, whp. Since $a(n)$ is chosen based on Eq. (2) and is greater than $100 \log n / n$, each cell has $\Theta(n a(n))$ nodes (by Lemma 3 ). Besides, each cell has $O(n \sqrt{a(n)})$ flows and Step 2(a) assigns nearly the same number of flows. So, Step 2(b) assigns to any node in the network at most $O(1 / \sqrt{a(n)})$ flows. Combining with Step $2(a)$, the total flows assigned to every node is $O(1+F(n)+1 / \sqrt{a(n)})$, which is also dominated by $O(1 / \sqrt{a(n)})$ (note that $a(n)$ is at most $(1 / F(n))^{2}$, hence $F(n)$ is at most $\left.1 / \sqrt{a(n)}\right)$.
Step 3 (Transmission Scheduling): We consider a scheduling scheme for a $(1, c)$-network. Any transmissions in this network must satisfy these two additional constraints simultaneously: 1) each interface only allows one transmission/reception at the same time, and 2) any two transmissions on any channel should not interfere with each other.

We propose a time-division multi-access (TDMA) scheme to schedule transmissions, which satisfy the above two constraints. In this scheme, a second is divided into a number of edge-color slots and at most one transmission/reception is scheduled at every node during each edge-color slot. Hence, the first constraint is satisfied. Each edge-color slot can be further split into smaller mini-slots. In each mini-slot, each transmission satisfies the above two constraints. Suppose that an omnidirectional antenna needs $c_{1}$ channels to separate $t_{1}$ concurrent transmissions. Intuitively, directional antennas can reduce the number of channels to $\left(\frac{\theta}{2 \pi}\right)^{2} c_{1}$ because directional antennas can separate the current transmissions if both the transmitter and the receiver use directional antennas. Thus, the number of mini-slots is reduced by a factor of $\left(\frac{\theta}{2 \pi}\right)^{2}$.

Then, we describe the two time slots as follows. Fig. 6 depicts a schedule of transmission on the network.

(i) Edge-color slot: First, we construct a routing graph in which vertices are the nodes in the network and an edge denotes transmission/reception of a node. In this construction, one hop along a flow is associated with one edge in the routing graph. In [8] and [22], it is shown that this routing graph can be edge-colored with at most $O(1 / \sqrt{a(n)})$ colors. Then, we divide one second into $O(1 / \sqrt{a(n)})$ edge-color slots and each slot has a length of $\Omega(\sqrt{a(n)})$ seconds. Each slot is stained with a unique edge-color. Since all edges connecting to a vertex use different colors, each node has at most one transmission/reception scheduled in any edge-color time slot.

(ii) Mini-slot: We further divide each edge-color slot into mini-slots. Then, we build a schedule that assigns a transmission to a node in a mini-slot within an edge-color slot over a channel. We construct an interference graph in which vertices are the nodes in the network and edges denote interference between two nodes. By Lemma 4, every cell has at most a constant number of interfering cells with a factor $\left(\frac{\theta}{2 \pi}\right)^{2}$, and each cell has $\Theta(n a(n))$ nodes (by Lemma 3). Thus, each node has at most $O\left(\left(\frac{\theta}{2 \pi}\right)^{2} n a(n)\right)$ edges in the interference graph. It is shown that a graph of degree at most $k$ can be vertex-colored with at most $k+1$ colors [22]. Hence, the interference graph can be vertex-colored with at most $O\left(\left(\frac{\theta}{2 \pi}\right)^{2} n a(n)\right)$ colors. Then, we use $k_{3}\left(\frac{\theta}{2 \pi}\right)^{2} n a(n)$ to denote the number of vertexcolors (where $k_{3}$ is a constant). Two nodes assigned the same vertex-color do not interfere with each other, while two nodes stained with different colors may interfere with each other. So, we need to schedule the interfering nodes either on different channels, or at different mini-slots on the same channel. We divide each edge-color slot into $\left\lceil\left(\frac{\theta}{2 \pi}\right)^{2} \frac{k_{3} n a(n)}{c}\right\rceil$ mini-slots on every channel, and assign the mini-slots on each channel from 1 to $\left[\left(\frac{\theta}{2 \pi}\right)^{2} \frac{k_{3} n a(n)}{c}\right]$. A node assigned with a color $s$, $1 \leq s \leq\left(\frac{\theta}{2 \pi}\right)^{2} k_{3} n a(n)$ is allowed to transmit in mini-slot $\left\lceil\frac{s}{c}\right\rceil$ 


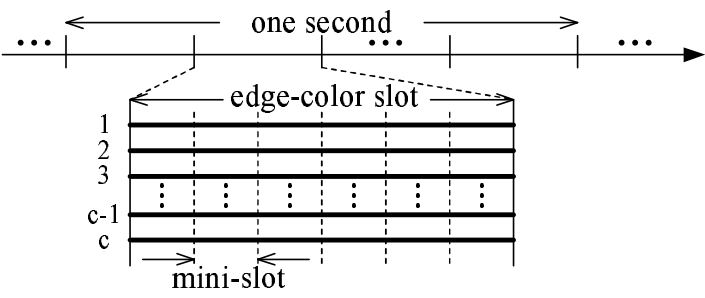

Fig. 6. The TDMA transmission schedule

on channel $(s \bmod c)+1$.

Let us analyze the capacity of the $(1, c)$-network. Each edge-color slot has a length of $\Omega(\sqrt{a(n)})$ seconds. Each edge-color slot is divided into $\left[\left(\frac{\theta}{2 \pi}\right)^{2} \frac{k_{3} n a(n)}{c}\right]$ mini-slots over every channel. Therefore, each mini-slots has a length of $\Omega\left(\frac{\sqrt{a(n)}}{\left[\left(\frac{\theta}{2 \pi}\right)^{2} \frac{k_{3} n a(n)}{c}\right\rceil}\right)$ seconds. Since each channel can transmit at the rate of $\frac{W}{c}$ bits/second, in each mini-slot, $\lambda(n)=\Omega\left(\frac{W \sqrt{a(n)}}{c\left\lceil\left(\frac{\theta}{2 \pi}\right)^{2} \frac{k_{3} n a(n)}{c}\right\rceil}\right)$ bits can be transported. Since $\left\lceil\left(\frac{\theta}{2 \pi}\right)^{2} \frac{k_{3} n a(n)}{c}\right\rceil \leq\left(\frac{\theta}{2 \pi}\right)^{2} \frac{k_{3} n a(n)}{c}+1$, we have, $\lambda(n)=\Omega\left(\frac{W \sqrt{a(n)}}{\left(\frac{\theta}{2 \pi}\right)^{2} k_{3} n a(n)+c}\right)$ bits/sec. Hence, $\lambda(n)=$ $\Omega\left(M I N_{O}\left(\frac{W}{\left(\frac{\theta}{2 \pi}\right)^{2} n \sqrt{a(n)}}, \frac{W \sqrt{a(n)}}{c}\right)\right)$ bits/sec. Since each flow is scheduled in one mini-slot on each hop during one second interval and every source-destination flow can support a per-node throughput of $\lambda(n)$ bits/sec, within one second, there are $\Omega\left(M I N_{O}\left(\frac{W}{\left(\frac{\theta}{2 \pi}\right)^{2} \sqrt{a(n)}}, \frac{W n \sqrt{a(n)}}{c}\right)\right)$ bits transmitted. Thus, the network capacity is $n \lambda(n)=$ $\Omega\left(M I N_{O}\left(\frac{W}{\left(\frac{\theta}{2 \pi}\right)^{2} \sqrt{a(n)}}, \frac{W n \sqrt{a(n)}}{c}\right)\right)$ bits/sec.

Then, we extend the result to an $(m, c)$ network, and the capacity of an $(m, c)$-network is $\Omega\left(M I N_{O}\left(\frac{W}{\left(\frac{\theta}{2 \pi}\right)^{2} \sqrt{a(n)}}, \frac{W m n \sqrt{a(n)}}{c}\right)\right)$. From Eq. (2), the size of each cell is $\min \left(\max \left(\frac{100 \log n}{n}, \frac{c}{n}\left(\frac{\theta}{2 \pi}\right)^{2}\right),\left(\frac{1}{F(n)}\right)^{2}\right)$, where $F(n)=\Theta\left(\frac{\log n}{\log \log n}\right)$. Substituting the three values, we have the following theorem.

Theorem 5: The constructive lower bound on the capacity of an $(m, c)$-network is as follows.

1) When $\frac{c}{m}$ is $O\left(\left(\frac{\theta}{2 \pi}\right)^{2} \log n\right), a(n)=\Theta\left(\frac{\log n}{n}\right)$, the network capacity is $\Omega\left(\frac{W}{\theta^{2}} \sqrt{\frac{n}{\log n}}\right)$ bits/sec.

2) When $\frac{c}{m}$ is $\Omega\left(\left(\frac{\theta}{2 \pi}\right)^{2} \log n\right)$ and also $O\left(\left(\frac{\theta}{2 \pi}\right)^{2} n\left(\frac{\log \log n}{\log n}\right)^{2}\right)$ and $a(n)=\Theta\left(\frac{c \theta^{2}}{m n}\right)$, the network capacity is $\Omega\left(\frac{W}{\theta} \sqrt{\frac{n m}{c}}\right)$.

3) When $\frac{c}{m}$ is $\Omega\left(\left(\frac{\theta}{2 \pi}\right)^{2} n\left(\frac{\log \log n}{\log n}\right)^{2}\right)$ and $a(n)=$ $\Theta\left(\left(\frac{\log \log n}{\log n}\right)^{2}\right)$, the network capacity is $\Omega\left(\frac{W m n \log \log n}{c \log n}\right)$ bits/sec.

\section{Some Possible Implications}

Using directional antennas in multi-channel networks can improve the network capacity by enhancing the connectivity and reducing interference. When $\frac{c}{m}$ is $O\left(\left(\frac{\theta}{2 \pi}\right)^{2} \log n\right)$, the capacity is $\Theta\left(\frac{W}{\theta^{2}} \sqrt{\frac{n}{\log n}}\right)$, which is obtained under the connectivity constraint. This capacity has a capacity gain $\frac{4 \pi^{2}}{\theta^{2}}$ over an MC-MOmni network. This result implies that directional antennas can greatly improve the network connectivity. When $\frac{c}{m}$ is increased to $\Omega\left(\left(\frac{\theta}{2 \pi}\right)^{2} \log n\right)$, the throughput capacity degrades to $\Theta\left(\frac{W}{\theta} \sqrt{\frac{n m}{c}}\right)$. At that time, directional antennas can significantly mitigate the interference and the network has a capacity gain of $\frac{2 \pi}{\theta}$.

On the other hand, using multiple channels can help to solve the problems of hidden terminals and deafness caused by directional antennas. The directional hidden terminal problem happens when a transmitter fails to hear a prior RTS/CTS exchange between another pair of nodes and cause collisions by initiating a transmission to the receiver of the ongoing transmission. The deafness problem occurs when a transmitter fails to communicate to its intended receiver, because the receiver's antenna is adjusted in a different direction. Elbatt et al. [23] solved the deafness problem by using two interfaces which are tuned to two different channels. Both the hidden terminal and deafness problems were mitigated by sending busy tones over another channel from an omnidirectional antenna [24]. Thus, integrating multiple channels with directional antennas can improve the network performance further.

\section{CONCLUSION}

Previous studies [2]-[7] focused on using multiple channels in wireless networks to improve the network performance. However, since only omnidirectional antennas are equipped at every node in such networks, the improvement on the network capacity is limited by high interference. Other studies [9]-[16] found that using directional antennas instead of omnidirectional antennas in networks can greatly improve the network capacity. But, such single-channel networks using directional antennas only allow limited concurrent transmissions.

In this paper, we propose a novel wireless network that integrates multi-channel and directional antennas. We derive the upper bounds and lower bounds on the capacity under arbitrary networks and random networks. We have found that using directional antennas in multi-channel networks not only can enhance network connectivity but also can mitigate interferences. Meanwhile, using multiple channels also helps to solve the hidden terminal and deaf problems [12] caused by directional antennas. Therefore, combining multiple channels with directional antennas can achieve significant improvement on the network performance.

\section{ACKNOWLEDGMENT}

This research was partially supported by Natural Science Foundation of China grant No.60573138 and 60773091, and the National Grand Fundamental Research 973 Program of China under Grant No.2006CB303000.

\section{REFERENCES}

[1] P. Gupta and P. R. Kumar, "The capacity of wireless networks," IEEE Transactions on Information Theory, vol. 46, no. 2, pp. 388 - 404, 2000. 
[2] A. Raniwala and T. Chiueh, "Architecture and algorithms for an IEEE 802.11-based multi-channel wireless mesh network," in INFOCOM, 2005.

[3] J. So and N. H. Vaidya, "Multi-channel MAC for ad hoc networks: Handling multi-channel hidden terminals using a single transceiver,' in MobiHoc, 2004

[4] P. Kyasanur and N. H. Vaidya, "Routing and interface assignment in multi-channel multi-interface wireless networks," in WCNC, 2005.

[5] A. Nasipuri, J. Zhuang, and S. Das, "A multichannel CSMA MAC protocol for multihop wireless networks," in WCNC, 1999.

[6] P. Bahl, R. Chandra, and J. Dunagan, "SSCH: Slotted seeded channel hopping for capacity improvement in ieee 802.11 ad-hoc wireless networks," in Mobicom, 2004.

[7] R. Draves, J. Padhye, and B. Zill, "Routing in multi-radio, multi-hop wireless mesh networks," in Mobicom, 2004.

[8] P. Kyasanur and N. H. Vaidya, "Capacity of multichannel wireless networks: Impact of number of channels and interfaces," in MobiCom, 2005.

[9] S. Yi, Y. Pei, and S. Kalyanaraman, "On the capacity improvement of ad hoc wireless networks using directional antennas," in MobiHoc, 2003.

[10] R. Ramanathan, "On the performance of ad hoc networks with beamforming antennas," in MobiHoc, 2001

[11] M. Takai, J. Martin, R. Bagrodia, and A. Ren, "Directional virtual carrier sensing for directional antennas in mobile ad hoc networks," in MobiHoc, 2002.

[12] R. R. Choudhury, X. Yang, N. H. Vaidya, and R. Ramanathan, "Using directional antennas for medium access control in ad hoc networks," in MOBICOM, 2002

[13] L. Bao and J. Garcia-Luna-Aceves, "Transmission scheduling in ad hoc networks with directional antennas," in MOBICOM, 2002.

[14] T. Korakis, G. Jakllari, and L. Tassiulas, "A MAC protocol for full exploitation of directional antennas in ad-hoc wireless networks," in MobiHoc, 2003

[15] Z. Zhang, "Pure directional transmission and reception algorithms in wireless ad hoc networks with directional antennas," in IEEE ICC, 2005.

[16] R. Ramanathan, J. Redi, C. Santivanez, D. Wiggins, and S. Polit, "Ad hoc networking with directional antennas: A complete system solution," IEEE JSAC, vol. 23, no. 3, pp. 496-506, 2005.

[17] S. M. Das, H. Pucha, D. Koutsonikolas, Y. C. Hu, and D. Peroulis, "DMesh: Incorporating practical directional antennas in multi-channel wireless mesh networks," IEEE JSAC special issue on Multi-Hop Wireless Mesh Networks, vol. 24, 2006.

[18] J. Zhang and S. C. Liew, "Capacity improvement of wireless ad hoc networks with directional antennae," in IEEE VTC, 2006-Spring.

[19] H.-N. Dai, K.-W. Ng, and M.-Y. Wu, "On collision-tolerant transmission with directional antennas," in IEEE WCNC, 2008.

[20] M. Raab and A. Steger, "Balls into bins - A simple and tight analysis," Lecture Notes in Computer Science, vol. 1518, pp. 159-170, 1998.

[21] A. Gamal, J. Mammen, B. Prabhakar, and D. Shah, "Throughput-delay trade-off in wireless networks," in INFOCOM, 2004.

[22] D. B. West, Introduction to graph theory, 2nd ed. Upper Saddle River, N.J.: Prentice Hall PTR, 2001.

[23] T. ElBatt, T. Anderson, and B. Ryu, "Performance evaluation of multiple access protocols for ad hoc networks using directional antennas," in IEEE WCNC, 2003

[24] H.-N. Dai, K.-W. Ng, and M.-Y. Wu, "A busy-tone based MAC scheme for wireless ad hoc networks using directional antennas," in IEEE Globecom, 2007.

\section{APPENDIX A}

Proof of Theorem 1: First, we consider a unit-area plane which has $n$ nodes arbitrarily placed. There are $c$ channels available in the network. The whole network transports $\lambda n T$ bits over $T$ seconds. Let the average distance between the source node and the destination node of a bit be $\bar{L}$. Thus, a transport capacity of $\lambda n \bar{L}$ bit-meters per second is achieved.

Let us consider bit $b$, where $1 \leq b \leq \lambda n T$. Suppose that bit $b$ moves from its source to its destination in a sequence of $h(b)$ hops, where the $h$-th hop traverses a distance of $r_{b}^{h}$. Then, we have

$$
\lambda n T \bar{L} \leq \sum_{b=1}^{\lambda n T} \sum_{h=1}^{h(b)} r_{b}^{h}
$$

We define $H$ to be the total number of hops traversed by all bits in $T$ seconds, i.e., $H=\sum_{b=1}^{\lambda n T} h(b)$. Therefore, the number of bits transmitted by all nodes within $T$ seconds is equal to $H$. Since each node has $m$ interfaces, and each interface transmits over a channel with rate $W / c$, the total number of bits that can be transmitted by all nodes over all interfaces is at most $\frac{W T n m}{2 c}$. Therefore, we have

$$
H=\sum_{b=1}^{\lambda n T} h(b) \leq \frac{W T n m}{2 c}
$$

It is shown in [8] that each hop consumes a disk of radius $\frac{\Delta}{2}$ times the length of the hop around each receiver, i.e., $\frac{\Delta}{2} \cdot r_{b}^{h}$.

Meanwhile, from the second condition of Eq. (1), only when a node adjusts its beam toward a receiver and the receiver is only affected by the nodes within its antenna beam, as shown in Fig. 4. On average, $\frac{\theta}{2 \pi}$ proportion of the nodes inside the reception beam will interfere with the receiver. Thus, the conditional interference zone area is $\frac{\theta}{2 \pi}\left[\pi\left(\frac{\Delta}{2} r_{b}^{h}\right)^{2} \frac{\theta}{2 \pi}\right]=\frac{\Delta \theta^{2}}{16 \pi}\left(r_{b}^{h}\right)^{2}$

Then, we have the constraint $\sum_{b=1}^{\lambda n T} \sum_{h=1}^{h(b)} \frac{\Delta^{2} \theta^{2}}{16 \pi}\left(r_{b}^{h}\right)^{2} \leq W T$, which can be rewritten as

$$
\sum_{b=1}^{\lambda n T} \sum_{h=1}^{h(b)} \frac{1}{H}\left(r_{b}^{h}\right)^{2} \leq \frac{16 \pi W T}{\Delta^{2} \theta^{2} H}
$$

Since the quadratic function is convex, we have

$$
\left(\sum_{b=1}^{\lambda n T} \sum_{h=1}^{h(b)} \frac{1}{H} r_{b}^{h}\right)^{2} \leq \sum_{b=1}^{\lambda n T} \sum_{h=1}^{h(b)} \frac{1}{H}\left(r_{b}^{h}\right)^{2}
$$

Therefore, combining (5) and (6) yields

$$
\sum_{b=1}^{\lambda n T} \sum_{h=1}^{h(b)} r_{b}^{h} \leq \sqrt{\frac{16 \pi W T H}{\Delta^{2} \theta^{2}}}
$$

Then, substituting (4) in (7) gives

$$
\sum_{b=1}^{\lambda n T} \sum_{h=1}^{h(b)} r_{b}^{h} \leq \frac{W T}{\Delta \theta} \sqrt{\frac{8 \pi n m}{c}}
$$

Finally, we substitute (3) in (8), and obtain

$$
\lambda n \bar{L} \leq \frac{W}{\Delta \theta} \sqrt{\frac{8 \pi n m}{c}}
$$

This proves that the network capacity of an arbitrary network is $O\left(\frac{W}{\theta} \sqrt{\frac{n m}{c}}\right)$ bit-meters/sec. Compared with the result in [8], i.e., the capacity of an MC-MOmni network is $\frac{W}{\Delta} \sqrt{\frac{2 n m}{\pi c}}$, an MC-MDA network has a capacity gain of $\frac{2 \pi}{\theta}$.

\section{APPENDIX B}

Proof of Lemma 4: Suppose that there is a cell D that can transmit with its 8 neighboring cells. The transmission range of each node in cell $\mathrm{D}, r(n)$, is defined as the distance between the transmitter and the receiver. Since each cell has the size $a(n), r(n)$ is no more than $3 \sqrt{a(n)}$ (if including the cell itself, there are 9 cells).

From the interference model Eq. (1), the transmission is successful only when the interfering nodes are $(1+\Delta) r(n)$ away from the receiver or the interfering nodes will not cause interference at the receiver (the beams of the interfering nodes do not cover the receiver). Let us consider that a transmitter $X_{i}$ within cell $\mathrm{B}$ is transmitting a data packet to a receiver $X_{j}$ within cell A. Since the transmission range between $X_{i}$ and $X_{j}$ is $r(n)$, the distance between two transmitter $X_{k}$ and $X_{i}$ must be less than $(2+\Delta) r(n)$, if $X_{k}$ causes the interference with $X_{j}$. Thus, an interfering area is loosely bounded within a square with an edge length of $3(2+\Delta) r(n)$.

Meanwhile, to ensure a successful transmission, the beams of the two nodes are pointed at each other. Therefore, only the nodes within the receiving beam of $X_{j}$ can interfere with the reception at $X_{j}$. Furthermore, only when a transmitter adjusts its beam to the receiver, it can interfere with the receiver. Therefore, the interfering probability is $\left(\frac{\theta}{2 \pi}\right)^{2}$.

Combining the two observations, there are at most $k_{2}=$ $\frac{(3(2+\Delta) r(n))^{2}}{a(n)} \cdot\left(\frac{\theta}{2 \pi}\right)^{2}=81(2+\Delta)^{2} \frac{\theta^{2}}{4 \pi^{2}}$ interfering cells. Hence, the number of interfering cells is bounded by $81(2+\Delta)^{2} \frac{\theta^{2}}{4 \pi^{2}}$, which is a constant $k_{2}$ independent of $a(n)$ and $n$. 